\title{
In silico analysis of maize HDACs with an emphasis on their response to biotic and abiotic stresses
}

\author{
Kang Zhang Equal first author, 1, ${ }^{2}$, Lu Yu ${ }^{\text {Equal first author, } 1,2}$, Xi Pang ${ }^{1,2}$, Hongzhe Cao ${ }^{1,2}$, Helong Si ${ }^{1,2}$, Jinping Zang ${ }^{1,2}$, Jihong \\ Xing ${ }^{\text {Corresp., } 1,2}$, Jingao Dong ${ }^{\text {Corresp. 1, } 2}$ \\ ${ }^{1}$ College of Life Science, Hebei Agricultrual University, Baoding, Hebei, China \\ 2 Hebei Key Laboratory of Plant Physiology and Molecular Pathology, Hebei Agricultrual University, Baoding, Hebei, China \\ Corresponding Authors: Jihong Xing, Jingao Dong \\ Email address: xingjihong@hebau.edu.cn, dongjingao@126.com
}

Histone deacetylases (HDACs) are key epigenetic factors in regulating chromatin structure and gene expression in multiple aspects of plant growth, development, and response to abiotic or biotic stresses. Many studies on systematic analysis and molecular function of HDACs in Arabidopsis and rice have been conducted. However, systematic analysis of HDAC gene family and gene expression in response to abiotic and biotic stresses has not yet been reported. In this study, a systematic analysis of the HDAC gene family in maize was performed and 18 ZmHDACs distributed on 9 chromosomes were identified. Phylogenetic analysis of ZmHDACs showed that this gene family could be divided into RPD3/HDA1, SIR2, and HD2 groups. Tissue-specific expression results revealed that ZmHDACs exhibited diverse expression patterns in different tissues, indicating that these genes might have diversified functions in growth and development. Expression pattern of ZmHDACs in hormone treatment and inoculation experiment suggested that several ZmHDACs might be involved in jasmonic acid or salicylic acid signaling pathway and defense response. Interestingly, HDAC genes were downregulated under heat stress, and immunoblotting results demonstrated that histones H3K9ac and H4K5ac levels were increased under heat stress. These results provide insights into ZmHDACs, which could help to reveal their functions in controlling maize development and responses to abiotic or biotic stresses. 
1 In silico analysis of maize HDACs with an emphasis on their response to biotic

2

3

4 Kang Zhang ${ }^{\#, 1,2}$, Lu Yu ${ }^{\#, 1,2}$, Xi Pang ${ }^{1,2}$, Hongzhe Cao ${ }^{1,2}$, Helong Si ${ }^{1,2}$, Jinping Zang ${ }^{1,2}$, Jihong

$5 \quad$ Xing $*, 1,2$, Jingao Dong*,1,2

6

$7 \quad{ }^{1}$ College of Life Science, Hebei Agricultural University, Baoding, 071000, China; ${ }^{2}$ Hebei Key

8 Laboratory of Plant Physiology and Molecular Pathology, Hebei Agricultural University, Baoding,

9 071000, China

10

11

12

13

14

15

16

17

18

19 Jingao Dong

20 E-mail: dongjingao@126.com

21 Tel.: +86-312-7528266

22 Fax: $+86-312-7528266$

\section{and abiotic stresses}

* Authors for correspondence

Jihong Xing

E-mail: xingjihong2000@126.com

Tel.: +86-312-7528142 
24

25

26

27

\section{Abstract}

Histone deacetylases (HDACs) are key epigenetic factors in regulating chromatin structure and gene expression in multiple aspects of plant growth, development, and response to abiotic or biotic stresses. Many studies on systematic analysis and molecular function of HDACs in Arabidopsis and rice have been conducted. However, systematic analysis of HDAC gene family and gene expression in response to abiotic and biotic stresses has not yet been reported. In this study, a systematic analysis of the HDAC gene family in maize was performed and 18 ZmHDACs distributed on 9 chromosomes were identified. Phylogenetic analysis of ZmHDACs showed that this gene family could be divided into RPD3/HDA1, SIR2, and HD2 groups. Tissue-specific expression results revealed that ZmHDACs exhibited diverse expression patterns in different tissues, indicating that these genes might have diversified functions in growth and development. Expression pattern of ZmHDACs in hormone treatment and inoculation experiment suggested that several ZmHDACs might be involved in jasmonic acid or salicylic acid signaling pathway and defense response. Interestingly, HDAC genes were downregulated under heat stress, and immunoblotting results demonstrated that histones $\mathrm{H} 3 \mathrm{~K} 9 \mathrm{ac}$ and $\mathrm{H} 4 \mathrm{~K} 5 \mathrm{ac}$ levels were increased under heat stress. These results provide insights into ZmHDACs, which could help to reveal their functions in controlling maize development and responses to abiotic or biotic stresses.

Keywords: maize, histone deacetylase, phylogenetic relationship, expression, stress response 


\section{Introduction}

Dynamic chromatin structures have primary importance in modulating gene activities in higher eukaryotes (Luger et al. 2012). The chromatin structure can be affected by histone modifications, DNA methylation, and chromatin remodeling (Allis \& Jenuwein 2016). Among histone modifications, histone acetylation is one of the most widely studied (Shahbazian \& Grunstein 2007). Histone acetylation is generally associated with a chromatin structure that is open and therefore accessible to transcription factors or transcription regulators (Liu et al. 2014). The level of histone acetylation is regulated by histone acetyltransferases (HATs) and histone deacetylases (HDACs) (Peserico \& Simone 2011).

HDACs are a class of enzymes that remove acetyl groups from core histones $(\mathrm{H} 2 \mathrm{~A}, \mathrm{H} 2 \mathrm{~B}, \mathrm{H} 3$, and H4), thereby regulating gene expression. They are widely distributed in animals, yeasts, and plants. Based on homology analysis of yeast HDACs sequences, HDACs in plants can be grouped into three different families: the RPD3/HDA1, SIR2, and HD2 family (Pandey et al. 2002). RPD3/HDA1 is the largest family of HDACs, with members that are homologous to yeast RPD3 and HDA1 and contain a typical HDAC domain (Yang \& Seto 2008). The members of the SIR2 family are conserved from prokaryotes to eukaryotes, and use $\mathrm{NAD}^{+}$as a coenzyme to exercise HDACs activity (Imai et al. 2000). HD2 family members were first identified in maize and have not yet been detected in yeasts and animals (Lusser et al. 1997). In Arabidopsis, HD2 family members have a conserved terminal amino acid region (EFWG motif), and HDT1 and HDT3 contain a $\mathrm{C} 2 \mathrm{H} 2$ type zinc finger domain, which may mediate DNA-protein or protein-protein interactions (Zhou et al. 2004).

A total of 18 HDACs have been identified in the genome of Arabidopsis, which belong to three aforementioned HDAC families (Pandey et al. 2002). In recent years, increasing evidences show that HDACs play important roles in regulating epigenetic processes in Arabidopsis in response to abiotic and biotic stresses. $H D A 6$ and $H D A 19$ are involved in abscisic acid (ABA) signaling pathway, and can be induced by jasmonic acid (JA) and ethylene (ET) (Chen et al. 2010; Chen \& Wu 2010). HDA6 can interact with EIN3 and JAZ proteins, and is involved in ET and JA 
71

72

73

74

signaling pathways (Zhu et al. 2011), while HDA19 participates in salicylic acid (SA)-mediated plant defense responses and regulates PRI (Pathogenesis Related 1) expression by inhibiting WRKY38 and WRKY62, which encode two structurally related type III WRKY transcription factors (Kim et al. 2008). In hdal9 mutant, genes related to SA signaling pathway have been observed to be overexpressed and $P R$ genes have been demonstrated to show increased expression. Besides, the mutant has also been reported to exhibit enhanced resistance to Pseudomonas syringae pv tomato strain DC3000 (Choi et al. 2012). The HD2D gene has been noted to influence plant growth, development, and abiotic response (Han et al. 2016). In rice, OsHDACs play an important role in abiotic and biotic stresses. Both HDT701 and HDT705 have been observed to be localized in the nucleus and involved in the regulation of seed germination in response to abiotic stresses (Zhao et al. 2014a; Zhao et al. 2016). Overexpression of HDT701 can decrease the level of histone $\mathrm{H} 4$ acetylation and enhance susceptibility to the rice pathogens Magnaporthe oryzae and Xanthomonas oryzae, indicating that HDT701 negatively regulates the innate immunity by modulating the levels of histone $\mathrm{H} 4$ acetylation of pattern recognition receptor and defense-related genes (Ding et al. 2012).

In maize, ZmHDACs have been reported to regulate plant development, seed dormancy, and germination. Maize HDAC genes HDA101, HDA102, and HDA108 have been found to show similar expression levels in endosperm; for example, HDA108 controls vegetative and reproductive development and HDA101 influences seed development by regulating histone acetylation levels (Varotto et al. 2003; Rossi et al. 2007; Yang et al. 2016; Forestan et al. 2018). In addition, ZmHDACs activities are required for GA-induced programmed cell death in aleurone layers, and regulate programmed cell death via ROS-mediated signal transduction pathway (Hou et al. 2015; Hou et al. 2017). Nevertheless, systematic analysis of all the $H D A C$ genes and their responses to abiotic and biotic stresses in maize has not yet been reported. In the present study, the $H D A C$ gene family was identified from the maize genome, the evolutionary relationships between maize, Arabidopsis, and rice were determined, and the conserve domain and tissue specific and stress responsive expression profiles were further analyzed. Together, our results revealed the 
98 importance of $Z m H D A C$ genes in various aspects of plant development and response to abiotic or 99 biotic stress.

100

\section{Materials and method}

102

103

\section{Plant materials and treatments}

The maize inbred line B73 was grown in the experimental field in Baoding (Hebei Agricultural University, Hebei province, China). For methyl jasmonate (MeJA; Sigma, USA), SA (Thermo Fisher Scientific, USA) treatments, MeJA $(100 \mu \mathrm{M})$ and SA $(100 \mu \mathrm{M}$ were sprayed onto the entire seedlings at the V3 growth stage (Li et al. 2015; Wang et al. 2015). Cold and heat stresses were applied by growing the seedlings under control conditions in an incubator at $4^{\circ} \mathrm{C}$ or $42^{\circ} \mathrm{C}$ from 12:00 pm for $12 \mathrm{~h}$. The control treatment temperature was $25^{\circ} \mathrm{C}$.

\section{Identification of HDACs in maize}

The maize genetics and genomics database (MaizeGDB, https://www.maizegdb.org) were searched to identify HDACs using BLASTP with a standard e-value $<1 \mathrm{e}-5$, with Arabidopsis and rice HDAC protein sequences as queries. Furthermore, the domains of all protein sequences were analyzed using Hidden Markov Model (HMM) of Pfam (http://pfam.sanger.ac.uk/search) and SMART database (http://smart.embl-heidelberg.de). The HDACs in maize in online server ExPASy were examined using bioinformatics software ExPASy-ProtParam tool (http://web.expasy.org/protparam/).

\section{Phylogenetic analysis}

ClustalW with default parameters was employed for multiple alignment of all HDAC protein sequences of maize, rice, and Arabidopsis (Larkin et al. 2007). The results of multiple alignment were imported to MEGA7.0 software for phylogenetic analysis, with neighbor-joining (NJ) method and 1000 bootstrap re-samplings (Kumar et al. 2016). 
125

126

127

128

129

130

\section{1}

132

133

134

135

136

137

138

139

140

141

142

143

144

145

146

147

150

151 The nuclei from leaf tissue in maize V3 stage were purified by sucrose density gradient

\section{Conserved domain analysis}

Protein sequences of HDAC family genes were obtained from maize genomics database. The conserved domain of maize HDACs was identified by SMART (http://smart.embl-heidelberg.de) and Pfam (http://pfam.xfam.org) with default parameters, and plotted by IBS software (Liu et al. 2015).

\section{Expression analysis of HDACs in maize}

Published transcriptome datasets were downloaded from NCBI Short Read Archive database (SRA). The RNA-Seq datasets (Table S2) were mapped to the reference genome of maize (B73 RefGen_V3) using TopHat with default parameters (Trapnell et al. 2009). Cufflinks software was used to calculate the expression levels using default parameters (Trapnell et al. 2012). The gene expression levels were normalized by gene length and read numbers to calculate FPKM (fragments per kilobase of transcript per million mapped reads) values. Heatmaps of maize HDACs expression levels were constructed by using Heml software (Deng et al. 2014).

\section{RNA isolation and quantitative real-time PCR}

All samples were homogenized in liquid nitrogen before RNA isolation. Total RNA was isolated using TRIZOL ${ }^{\circledR}$ reagent (Invitrogen, USA) and purified with Qiagen RNeasy columns (Qiagen, Germany). Quantitative real-time PCR (qRT-PCR) was conducted using actin as an internal reference and cDNAs from samples collected at different time points as template with TransStart Tip Green qPCR SuperMix according to the manufacturer's instructions. Furthermore, comparative $\mathrm{Ct}$ analysis (2- $\Delta \Delta \mathrm{Ct})$ of each gene in inbred $\mathrm{B} 73$ and its relative expression levels at different time points was employed, and quantitative data were expressed as mean \pm standard error of mean (SEM). The primer sequences used in this study are listed in Table S1.

\section{Immunoblotting analysis}


152

153

154

155

156

157

158

159

160

161

162

163

164

165

166

167

168

169

170

171

172

173

174

175

176

177

178

centrifugation, and nuclear proteins were isolated by protein extraction buffer. The nuclear proteins mixed with loading buffer were boiled for $5 \mathrm{~min}$. Then, the proteins were separated by $12 \%$ SDS-PAGE and the separated proteins were blotted onto PVDF membranes (Millipore, 0.22 $\mu \mathrm{m})$. The membranes were blocked (5\% milk dissolved in $1 \times$ TBST) at room temperature for $2 \mathrm{~h}$, and incubated overnight at $4{ }^{\circ} \mathrm{C}$ with anti-H3 (Abcam, ab1791), anti-H3K9ac (Abcam, ab10812), anti-H4K5ac (Millipore, 07-327), and anti-H4K8ac (Abcam, ab15823) antibodies. The membranes were washed thrice with TBST for $10 \mathrm{~min}$ and incubated for $2 \mathrm{~h}$ at room temperature with horseradish peroxidase labelled secondary antibodies. The membranes were washed three times with TBST, incubated in ECL for $1 \mathrm{~min}$, and examined using X-OMAT BT film in darkroom.

\section{Results}

\section{Identification and phylogenetic analysis of HDACs in maize}

The protein sequences of AtHDACs and OsHDACs were used as queries to conduct sequence homology searches against the maize genomics database. The length of complete coding sequence (CDS) and number of amino acids encoding the ZmHDAC family genes were obtained from the maize genomics database. In total, 18 independent maize HDAC family genes were identified (Table 1). The CDS of the retrieved ZmHDAC genes ranged from 501 to $2103 \mathrm{bp}$, while predicted proteins ranged from 166 to 700 amino acids with calculated molecular weights from 18.91 to $76.54 \mathrm{kDa}$ and isoelectric points from 4.61 to 9.31 (Table 1).

To evaluate the evolutionary relationship of plant HDACs, phylogenetic analysis was performed using the protein sequences of HDACs from maize, rice, and Arabidopsis. The phylogenetic tree indicated that the 18-uncovered maize HDACs could be grouped into three types characterized by distinctive protein structures (Figure 1). In maize, RPD3/HDA1 family HDACs consisted of 12 members based on their sequence similarity, all of which exhibited a characteristic HDAC domain (Figure 2) and could be further divided into three classes based on sequence similarity. Class I, II, and III included 6 HDACs, 5 HDACs, and 1 HDAC, respectively (Figure 
179

180

181

182

183

184

185

186

187

188

189

190

191

192

193

194

195

196

197

198

199

200

201

202

203

204

205

1). The phylogenetic analysis also demonstrated that maize has two SIR2 family HDACs with highly conserved Sir2 domains. Finally, four plant-specific HDACs (HD2 family) were identified, which might indicate that this protein has high DNA-binding affinity or could mediate proteinprotein interactions.

\section{Tissue-specific expression profiles of $H D A C$ genes in maize}

To investigate tissue- or organ-specific expression profiles of $H D A C$ genes in maize, transcriptome data from the SRA database were used, which included 22 different tissues or organs (Sekhon et al. 2014). As indicated in Figure 3, the expression patterns of the maize $H D A C$ genes could be divided into three clusters, cluster 1 , cluster 2 , and cluster 3 . Cluster 1 comprised two subgroups, C1-Sub1 and C1-Sub2, according to their expression levels. Genes in C1-Sub1 had a low expression level in anther and pollen. In contrast, genes in C1-Sub2 were highly expressed in anther and pollen, presenting an opposite expression trend, which indicated that these genes might be associated with reproductive growth. Genes in cluster 2 exhibited a low expression level in all the tissues investigated, especially GRMZM2G367886, GRMZM2G456473, and GRMZM5G807054. Genes in cluster 3 showed a higher expression level in root, stem, shoot apical meristem (SAM), seed, and endosperm, and had a lower expression level in anther and pollen, suggesting the possible involvement of these genes in cell differentiation and seed development.

\section{Expression profiles of $H D A C$ genes under biotic stress}

To explore the potential roles of ZmHDACs in biotic stress responses, we analyzed the expression pattern of HDAC family genes under Fusarium verticillioides infection from the public expression profile datasets (Shu et al. 2017). The results showed that the expression level of HDAC family genes presented different trends under $F$. verticillioides infection (Figure 4). For example, the expression levels of GRMZM2G057044 gradually decreased with the increase in treatment time. The expression level of GRMZM2G163572 was down regulated at 4 and 72 hpi (hours post infection), and up regulated at $48 \mathrm{hpi}$. The expression levels of GRMZM2G457889 were relatively 
206

207

208

209

210

211

212

213

214

215

216

217

218

219

220

221

222

223

224

225

226

227

228

229

230

231

232

stable before $48 \mathrm{hpi}$, but decreased at $72 \mathrm{hpi}$. In addition, GRMZM2G456473 was up regulated at 12 hpi, while GRMZM2G081474 was down regulated at $24 \mathrm{hpi}$.

\section{Expression profiles of $H D A C$ genes under abiotic stress}

It has been indicated that HDACs play important functions in response to abiotic stresses (Makarevitch et al. 2015). To explore the potential roles of ZmHDACs in abiotic stress responses, we treated maize seedlings with MeJA and SA, which are the most important stress-protective phytohormones, to determine the expression levels of ZmHDACs under hormones treatment. As shown in Figure 5, ZmHDAC genes significantly responded to MeJA treatments. Three HD2 family genes (GRMZM5G898314, GRMZM2G100146, and GRMZM2G159032) and three Class I family genes (GRMZM2G163572, GRMZM2G136067, and GRMZM2G367886) were downregulated. However, the expression levels of GRMZM2G172883, GRMZM2G046824, GRMZM2G056539, and GRMZM2G456473 were induced in 3 and $6 \mathrm{~h}$, but gradually decreased after $6 \mathrm{~h}$ of MeJA treatment. Under SA treatment (Figure 6), most of the ZmHDAC genes were downregulated, except a Class II family gene GRMZM2G046824, which was induced in 3 and $6 \mathrm{~h}$ of SA treatment. With regard to the downregulated genes under SA treatment, a fraction of the genes, such as GRMZM2G172883, GRMZM2G119703, GRMZM5G807054, GRMZM2G898314, GRMZM5G100146, and GRMZM2G057044, were downregulated in 3 and $6 \mathrm{~h}$ and upregulated in 12 and $24 \mathrm{~h}$ of SA treatment. These results suggested that differentially expressed genes might be involved in JA and SA signaling pathways.

Furthermore, we analyzed the transcriptome data under heat, UV, cold, salt, and drought stresses. The findings indicated that the maize $H D A C$ genes presented significant differential expression under abiotic stress (Figure 7). Under drought stress, most of the genes, such as GRMZM5G898314 and GRMZM2G119703, were significantly upregulated, while GRMZM2G457889 was downregulated. Similarly, under heat stress, most of the genes were downregulated, while GRMZM2G807054 and GRMZM2G172883 were upregulated. In addition, we analyzed the status of $\mathrm{H} 3$ acetylation following cold and heat treatments. The levels of $\mathrm{H} 3 \mathrm{~K} 9 \mathrm{ac}$ 
233

234

235

236

237

238

239

240

241

242

243

244

245

246

247

248

249

250

251

252

253

254

255

256

257

258

259

and H4K5ac decreased under cold treatment (Figure 8), but significantly increased after heat treatment, when compared with those in plants grown at normal temperatures. These findings suggested that maize HDACs might be involved in abiotic stress responses by regulating histone acetylation levels.

\section{Discussion}

HDACs, also known as lysine deacetylases (KDACs), are a class of enzymes that remove acetyl groups from core histones $(\mathrm{H} 2 \mathrm{~A}, \mathrm{H} 2 \mathrm{~B}, \mathrm{H} 3$, and $\mathrm{H} 4)$, thereby regulating gene expression (Makarevitch et al., 2015), and play critical roles in genome stability, plant growth and development, and response to environmental stresses (Luo et al. 2012a; Liu et al. 2014. Genomewide identification and characterization of HDACs have been reported in several plant species, such as Arabidopsis, Solanum lycopersicum, and Oryza sativa (Pandey et al. 2002; Fu et al. 2007; Zhao et al. 2014b). In maize, although HDAC genes have been identified (Demetriou et al. 2009), systematic analysis of the HDAC gene family in response to abiotic and biotic stresses is limited. In this study, 18 HDAC genes were identified in the maize genome, which belonged to three subfamilies: Class I (6), Class II (5), Class III (1), SRT (2), and HD2 (3). The number of ZmHDAC gene family in maize was found to be almost consistent with that in Arabidopsis, rice, and tomato, and gene expansion of HDACs was not obvious in maize (Pandey et al. 2002; Fu et al. 2007; Zhao et al. 2014b). In Class I, Class II, and Class III family, all members had a conventional HDAC conserved domain, and Sir2 and ZnF_C2H2 domains were identified in SRT and HD2 families. Two novel typical HDAC family members and one SRT family member were identified in the present study, which are important for research on HDACs in maize (Demetriou et al. 2009). The results of conserve domain analysis indicated that the function of HDAC family members in different plant species might have been stable during evolution, such as GRMZM2G136067 (HDA108) that can functionally complement a yeast $\operatorname{rpd} 3$ null mutant and influence the characteristic acetylation pattern on $\mathrm{H} 4$, suggesting a possible function for GRMZM2G136067 in the histone deposition process (Rossi et al. 1998; Kolle et al. 1999). 
260

261

262

263

264

265

266

267

268

269

270

271

272

273

274

275

276

277

278

279

280

281

282

283

284

285

286

In plants, HDACs are the key regulators of histone modification and chromatin remodeling, implying that epigenetic regulation play an important role in controlling gene expression in the developmental stages and responses to abiotic stress (Luo et al. 2012a; Ma et al. 2013; Liu et al. 2014). Accordingly, transcriptome data from public databases were further explored in the present study to dissect the expression profiles of the $\mathrm{ZmHDAC}$ genes. Several previous studies have shown that HDA6 is involved in plant growth and development, such as leaf development and flowering (Probst et al. 2004; Yu et al. 2011). GRMZM2G136067, an ortholog of HDA6, exhibited an expression pattern similar to GRMZM2G119703, with higher transcript accumulation during endosperm development (Varotto et al. 2003), suggesting that GRMZM2G136067 and GRMZM2G119703 might be involved in endosperm development. GRMZM2G172883, an ortholog of $H D A 19$, was highly expressed during seed development and germination, and several studies have confirmed that this gene plays an important role in the regulation of histone deacetylation during seed development (Rossi et al. 2007; Yang et al. 2016). The expression of GRMZM2G046824 and GRMZM2G56539, orthologs of HDA8 involved in sperm cell formation, was higher in anther and pollen, revealing that these two genes might be involved in flower development in maize. However, the tissues covered by the current datasets were limited, and tissue-specific analysis of ZmHDACs must include more types of tissues or datasets.

HDA6 and HDA19 can be induced by JA and ET (Zhou et al. 2005; Chen \& Wu 2010). HDA6 can interact with EIN3 and JAZ proteins, and is involved in ET and JA signaling pathways (Zhu et al. 2011). HDA19 participates in SA-mediated plant defense responses and regulates PRI expression by inhibiting the activity of WRKY38 and WRKY62 (Kim et al. 2008). The results of the present study revealed that several genes were induced in 3 and $6 \mathrm{~h}$, but gradually decreased after $6 \mathrm{~h}$ of MeJA treatment. With regard to SA treatment, several genes were gradually downregulated at 3 and $6 \mathrm{~h}$, and then upregulated at 12 and $24 \mathrm{~h}$ after the treatment. Disease resistance is regulated by several signal transduction pathways in which SA and JA function as key signaling molecules. In our results, several genes were induced or suppressed by JA and SA, which indicated that these genes might be involved in plant-pathogen interaction. For example, 
287 the expression pattern of GRMZM2G172883, an ortholog of HDA19 that can be induced by JA 288 (Zhou et al. 2005), was similar to that noted in Arabidopsis. In addition, the roles of HDA19 in 289 plant defense responses insinuated that GRMZM2G172883 might have some functions to regulate 290 plant immunity. These findings implied that the above-mentioned genes might be involved in 291 hormone signaling pathways and plant defense responses.

292 Several studies have demonstrated that HDACs are involved in plant resistance to abiotic 293 stress (Yuan et al. 2013). For instance, it has been reported that Arabidopsis overexpressing HD2C 294 exhibited greater tolerance to salt and drought stresses than the wild-type plants (Sridha \& Wu

295

296

297

2006). Besides, $H D 2 D$ can confer tolerance to abiotic stresses, including drought, salt, and cold stresses in Arabidopsis (Han et al. 2016), whereas HD2C can interact with HDA6 and regulate ABA-responsive gene expression by histone deacetylation (Luo et al. 2012b). In rice, several RPD3-type HDACs have been reported to be repressed under high salt and drought treatments (Hu et al. 2009). In the present study, under salt stress, most of the $H D A C$ genes were downregulated, while GRMZM2G056539 and GRMZM2G172883 were upregulated. A similar phenomenon also occurred under heat treatment, with most of the $H D A C$ genes being downregulated. It has been demonstrated that the expression of HDACs and the levels of histones H4ac, H3K9ac, and H4K5ac were reduced by low temperature in maize (Hu et al. 2011). As a considerable number of $H D A C$ genes were downregulated under heat stress, we used immunoblotting analysis to demonstrate the changes in histone acetylation levels under heat stress. The expressions of histones H3K9ac and H4K 5ac were reduced under cold stress, whereas the histone acetylation levels of these genes were increased under heat treatment. These results indicated that histone acetylation may play pivotal roles in plant responses to both cold and heat stresses.

\section{Conclusion}

In summary, we performed comprehensive analyses of HDAC gene family in maize, and identified 18 HDAC genes that can be divided into RPD3/HDA1, SIR2, and HD2 families. The domain arrangement was considerably conserved among members in the same groups or subgroups. Some 
$314 \mathrm{ZmHDAC}$ genes showed significant tissue-specific expression, as noted in tissue-specific 315 expression profiles, suggesting that these genes might participate in different organ development. 316 Several $H D A C$ genes exhibited different expression trends following MeJA treatment, SA 317 treatment, and fungal infection, indicating that these genes might be involved in defense response. 318 Interestingly, our results indicated that global histone acetylation in maize was affected by heat 319 stress, revealing that HDACs might be involved in the response of maize to abiotic stress. These 320 findings suggest that ZmHDACs might be important for plant development and response to biotic 321 and abiotic stress. Nevertheless, further research is required to determine the function and 322 molecular mechanisms of ZmHDACs in plant responses to biotic and abiotic stresses, which could 323 provide tools for the improvement of maize productivity. 
326

327

328

329

330

331

332

333

334

335

336

337

338

339

340

341

342

\section{Acknowledgments}

This work was supported by the National Key Research and Development Program of China (2016YFD0300704), National Natural Science Foundation of China (31901864), Natural Science Foundation of Hebei Province (C2019204141), China Agriculture Research System (CARS-0225), and Starting Grant from Hebei Agricultural University (ZD201721).

\section{Authors' contribution}

$\mathrm{KZ}$, JX, and DG conceived and designed the experiments; KZ, LY, XP, and HC performed the experiments; KZ, LY, HS, and JZ analyzed the experimental data; KZ, LY, HS, HC, and JZ prepared the figures and/or tables; KZ, JX, and JD drafted the work or revised it critically for important content; and KZ, JX, and JD approved the final draft of the manuscript submitted for review and publication. All the authors have read and approved the final manuscript.

\section{Conflict of interests}

The authors declare no conflict of interests. 
343

344

345

346

347

348

349

350

351

352

353

354

355

356

357

358

359

360

361

362

363

364

365

366

367

368

369

\section{Figure Legends}

Figure 1. Phylogenetic relationship of HDAC gene family among maize, rice, and Arabidopsis. Multiple sequences alignment and phylogenetic tree construction were performed using MEGA7.0. The value at the nodes represents bootstrap values from 1000 replicates. Different groups are shown by different colors.

Figure 2. Phylogenetic analysis and domain architecture of ZmHDACs. Construction of phylogenetic tree based on ZmHDACs amino acid sequences. Conserved domains of ZmHDACs were identified by Pfam and SMART. Different domains are indicated by different colors. The lengths of the domains in each protein are proportional. The NJ phylogenetic tree of ZmHDACs protein sequences was constructed using 1000 bootstrap replicates by MEGA 7.0. HDAC, Histone deacetylase domain; Sir2, Sir2 catalytic domain; ZnF_C2H2, Zinc finger C2H2 type domain.

Figure 3. Heatmaps representing the expression profiles of $\mathrm{ZmHDAC}$ genes in several tissues. Tissue-specific expression patterns of ZmHDAC genes associated with 22 different tissues or organs. The color scale on the right indicates expression values, with blue denoting high expression level and yellow representing low expression level.

Figure 4. Expression patterns of $Z m H D A C$ genes under $F$. verticillioides infection. The color scale on the right indicates fold changes (inoculated/mock), with blue denoting high fold change and yellow showing low fold change.

Figure 5. Expression patterns of $Z m H D A C$ genes in seedling leaf under MeJA treatment. qRTPCR was performed using gene-specific primers. Data are the mean $\pm \mathrm{SEM}$ of three independent experiments. 
370

371

372

373

374

375

376

377

378

379

380

381

382

383

384

385

386

387

388

Figure 6. Expression patterns of $Z m H D A C$ genes in seedling leaf under SA treatment. qRT-PCR was performed using gene-specific primers. Data are the mean \pm SEM of three independent experiments.

Figure 7. Expression patterns of $\mathrm{ZmHDAC}$ genes under heat, cold, salt, UV, and drought stresses. The color scale on the right indicates expression values, with blue denoting high expression level and yellow representing low expression level.

Figure 8. Immunoblot for the detection of H3K9ac and H4K5ac levels in seedling of the B73 inbred line under cold and heat treatments. Immunoblot with anti-H3 antibody was used as a loading control. CK mean the control treatment temperature $\left(25^{\circ} \mathrm{C}\right)$, cold and heat represented $4^{\circ} \mathrm{C}$ and $42^{\circ} \mathrm{C}$ treatment.

Table 1. HDAC genes in maize.

Table S1. Primers used in this study.

Table S2. Public RNA-Seq datasets used in this study. 
390

391

392

393

394

395

396

397

398

399

400

401

402

403

404

405

406

407

408

409

410

411

412

413

414

415

416

\section{REFERENCE}

Allis CD, and Jenuwein T. 2016. The molecular hallmarks of epigenetic control. Nature Reviews Genetice 17:487-500 DOI 10.1038/nrg.2016.59.

Chen LT, Luo M, Wang YY, and Wu K. 2010. Involvement of Arabidopsis histone deacetylase HDA6 in ABA and salt stress response. Journal of Experimental Botany 61:3345-3353 DOI $10.1093 / \mathrm{j} x \mathrm{~b} / \mathrm{erq} 154$

Chen LT, and Wu K. 2010. Role of histone deacetylases HDA6 and HDA19 in ABA and abiotic stress response. Plant Signaling \& Behavior 5:1318-1320 DOI 10.4161/psb.5.10.13168

Choi SM, Song HR, Han SK, Han M, Kim CY, Park J, Lee YH, Jeon JS, Noh YS, and Noh B. 2012. HDA19 is required for the repression of salicylic acid biosynthesis and salicylic acidmediated defense responses in Arabidopsis. The Plant Journal 71:135-146 DOI 10.1111/j.1365-313X.2012.04977.x

Demetriou K, Kapazoglou A, Tondelli A, Francia E, Stanca MA, Bladenopoulos K, and Tsaftaris AS. 2009. Epigenetic chromatin modifiers in barley: I. Cloning, mapping and expression analysis of the plant specific HD2 family of histone deacetylases from barley, during seed development and after hormonal treatment. Physiologia Plantarum 136:358-368 DOI $10.1111 / \mathrm{j} .1399-3054.2009 .01236 . \mathrm{x}$

Deng W, Wang Y, Liu Z, Cheng H, and Xue Y. 2014. HemI: a toolkit for illustrating heatmaps. Plos One 9:e111988 DOI 10.1371/journal.pone.0111988

Ding B, Bellizzi Mdel R, Ning Y, Meyers BC, and Wang GL. 2012. HDT701, a histone H4 deacetylase, negatively regulates plant innate immunity by modulating histone $\mathrm{H} 4$ acetylation of defense-related genes in rice. The Plant Cell 24:3783-3794 DOI $10.1105 /$ tpc. 112.101972

Forestan C, Farinati S, Rouster J, Lassagne H, Lauria M, Dal Ferro N, and Varotto S. 2018. Control of maize vegetative and reproductive development, fertility, and rRNAs silencing by HISTONE DEACETYLASE 108. Genetics 208:1443-1466 DOI 10.1534/genetics.117.300625 
$417 \mathrm{Fu} \mathrm{W}, \mathrm{Wu}$ K, and Duan J. 2007. Sequence and expression analysis of histone deacetylases in rice.

418

419

420

421

422

423

424

425

426

427

428

429

430

431

432

433

434

435

436

437

438

439

440

441

442

443
Biochemical and Biophysical Research Communications 356:843-850 DOI 10.1016/j.bbrc.2007.03.010

Han Z, Yu H, Zhao Z, Hunter D, Luo X, Duan J, and Tian L. 2016. AtHD2D gene plays a role in plant growth, development, and response to abiotic stresses in Arabidopsis thaliana. Frontiers in Plant Science 7:310 DOI 10.3389/fpls.2016.00310

Hou HL, Wang P, Zhang H, Wen H, Gao F, Ma NJ, Wang Q, and Li LJ. 2015. Histone acetylation is involved in gibberellin-regulated sodCp gene expression in maize aleurone layers. Plant and Cell Physiology 56:2139-2149 DOI 10.1093/pcp/pcv126

Hou HL, Zheng XK, Zhang H, Yue MX, Hu Y, Zhou H, Wang Q, Xie CS, Wang P, and Li LJ. 2017. Histone deacetylase is required for GA-induced programmed cell death in maize aleurone layers. Plant Physiology 175:1484-1496 DOI 10.1104/pp.17.00953

Hu Y, Qin F, Huang L, Sun Q, Li C, Zhao Y, and Zhou DX. 2009. Rice histone deacetylase genes display specific expression patterns and developmental functions. Biochemical and Biophysical Research Communications 388:266-271 DOI 10.1016/j.bbrc.2009.07.162

Hu Y, Zhang L, Zhao L, Li J, He S, Zhou K, Yang F, Huang M, Jiang L, and Li L. 2011. Trichostatin A selectively suppresses the cold-induced transcription of the ZmDREB1 gene in maize. Plos One 6:e22132 DOI 10.1371/journal.pone.0022132

Imai S, Armstrong CM, Kaeberlein M, and Guarente L. 2000. Transcriptional silencing and longevity protein Sir2 is an NAD-dependent histone deacetylase. Nature 403:795-800 DOI $10.1038 / 35001622$

Kim KC, Lai Z, Fan B, and Chen Z. 2008. Arabidopsis WRKY38 and WRKY62 transcription factors interact with histone deacetylase 19 in basal defense. The Plant Cell 20:2357-2371 DOI 10.1105/tpc.107.055566

Kolle D, Brosch G, Lechner T, Pipal A, Helliger W, Taplick J, and Loidl P. 1999. Different types of maize histone deacetylases are distinguished by a highly complex substrate and site specificity. Biochemistry 38:6769-6773 DOI 10.1021/bi982702v

Peer] reviewing PDF | (2019:06:38864:3:0:NEW 25 Dec 2019) 
444 Kumar S, Stecher G, and Tamura K. 2016. MEGA7: molecular evolutionary genetics analysis

445

446

447

448

449

450

451

452

453

454

455

456

457

458

459

460

461

462

463

464

465

466

467

468

469

470 version 7.0 for bigger datasets. Molecular Biology and Evolution 33:1870-1874 DOI 10.1093/molbev/msw054

Larkin MA, Blackshields G, Brown NP, Chenna R, McGettigan PA, McWilliam H, Valentin F, Wallace IM, Wilm A, Lopez R, Thompson JD, Gibson TJ, and Higgins DG. 2007. Clustal $\mathrm{W}$ and Clustal $\mathrm{X}$ version 2.0. Bioinformatics 23:2947-2948 DOI 10.1093/bioinformatics/btm404

Lee KK, and Workman JL. 2007. Histone acetyltransferase complexes: one size doesn't fit all. Nature Reviews Molecular Cell Biology 8:284-295 DOI 10.1038/nrm2145

Li DY, Fu FY, Zhang HJ, and Song FM. 2015. Genome-wide systematic characterization of the bZIP transcriptional factor family in tomato (Solanum lycopersicum L.). BMC Genomics 16:771 DOI 10.1186/S12864-015-1990-6

Liu W, Xie Y, Ma J, Luo X, Nie P, Zuo Z, Lahrmann U, Zhao Q, Zheng Y, Zhao Y, Xue Y, and Ren J. 2015. IBS: an illustrator for the presentation and visualization of biological sequences. Bioinformatics 31:3359-3361 DOI 10.1093/bioinformatics/btv362

Liu X, Yang S, Zhao M, Luo M, Yu CW, Chen CY, Tai R, and Wu K. 2014. Transcriptional repression by histone deacetylases in plants. Molecular Plant 7:764-772 DOI $10.1093 / \mathrm{mp} / \mathrm{ssu} 033$

Luger K, Dechassa ML, and Tremethick DJ. 2012. New insights into nucleosome and chromatin structure: an ordered state or a disordered affair? Nature Reviews Molecular Cell Biology 13:436 DOI 10.1038/nrm3382

Luo M, Liu X, Singh P, Cui Y, Zimmerli L, and Wu K. 2012a. Chromatin modifications and remodeling in plant abiotic stress responses. Biochimica Biophysica Acta-biomembranes 1819:129-136 DOI 10.1016/j.bbagrm.2011.06.008

Luo M, Wang YY, Liu XC, Yang SG, Lu Q, Cui YH, and Wu KQ. 2012b. HD2C interacts with HDA6 and is involved in ABA and salt stress response in Arabidopsis. Journal of Experimental Botany 63:3297-3306 DOI 10.1093/jxb/ers059 
471 Lusser A, Brosch G, Loidl A, Haas H, and Loidl P. 1997. Identification of maize histone

472

473

474 deacetylase HD2 as an acidic nucleolar phosphoprotein. Science 277:88-91 DOI 10.1126/science. 277.5322 .88

Ma XJ, Lv SB, Zhang C, and Yang CP. 2013. Histone deacetylases and their functions in plants. Plant Cell Reports 32:465-478 DOI 10.1007/s00299-013-1393-6

Makarevitch I, Waters AJ, West PT, Stitzer M, Hirsch CN, Ross-Ibarra J, and Springer NM. 2015. Transposable elements contribute to activation of maize genes in response to abiotic stress. PLoS Genetics 11:e1004915 DOI 10.1371/journal.pgen.1004915

Pandey R, Muller A, Napoli CA, Selinger DA, Pikaard CS, Richards EJ, Bender J, Mount DW, and Jorgensen RA. 2002. Analysis of histone acetyltransferase and histone deacetylase families of Arabidopsis thaliana suggests functional diversification of chromatin modification among multicellular eukaryotes. Nucleic Acids Research 30:5036-5055 DOI $10.1093 / \mathrm{nar} / \mathrm{gkf6} 60$

Peserico A, and Simone C. 2011. Physical and functional HAT/HDAC interplay regulates protein acetylation balance. Journal of Biomedicine and Biotechnology 2011:371832 DOI $10.1155 / 2011 / 371832$

Probst AV, Fagard M, Proux F, Mourrain P, Boutet S, Earley K, Lawrence RJ, Pikaard CS, Murfett J, Furner I, Vaucheret H, and Scheid OM. 2004. Arabidopsis histone deacetylase HDA6 is required for maintenance of transcriptional gene silencing and determines nuclear organization of rDNA repeats. The Plant Cell 16:1021-1034 DOI 10.1105/tpc.018754

Rossi V, Hartings H, and Motto M. 1998. Identification and characterisation of an RPD3 homologue from maize (Zea mays L.) that is able to complement an rpd3 null mutant of Saccharomyces cerevisiae. Molecular and General Genetics 258:288-296 DOI $10.1007 / \mathrm{s} 004380050733$

Rossi V, Locatelli S, Varotto S, Donn G, Pirona R, Henderson DA, Hartings H, and Motto M. 2007. Maize histone deacetylase hda101 is involved in plant development, gene transcription, and sequence-specific modulation of histone modification of genes and 
498

499

500

501

502

503

504

505

506

507

508

509

510

511

512

513

514

515

516

517

518

519

520

521

522

523

524

repeats. The Plant Cell 19:1145-1162 DOI $10.1105 /$ tpc.106.042549

Sekhon RS, Briskine R, Hirsch CN, Myers CL, Springer NM, Buell CR, de Leon N, and Kaeppler SM. 2014. Maize gene atlas developed by RNA sequencing and comparative evaluation of transcriptomes based on RNA sequencing and microarrays. PLoS One 8:e61005 DOI 10.1371/journal.pone.0061005

Shahbazian MD, and Grunstein M. 2007. Functions of site-specific histone acetylation and deacetylation. Annual Review of Biochemistry 76:75-100 DOI 10.1146/annurev.biochem.76.052705.162114

Shu X, Livingston DP, Woloshuk CP, Payne GA. 2017. Comparative histological and transcriptional analysis of maize kernels infected with Aspergillus flavus and Fusarium verticillioides. Frontiers in Plant Science 8:2075 DOI 10.3389/fpls.2017.02075

Sridha S, and $\mathrm{Wu} \mathrm{K}$. 2006. Identification of AtHD2C as a novel regulator of abscisic acid responses in Arabidopsis. The Plant Journal 46:124-133 DOI 10.1111/j.1365313X.2006.02678.x

Trapnell C, Pachter L, and Salzberg SL. 2009. TopHat: discovering splice junctions with RNASeq. Bioinformatics 25:1105-1111 DOI 10.1093/bioinformatics/btp120

Trapnell C, Roberts A, Goff L, Pertea G, Kim D, Kelley DR, Pimentel H, Salzberg SL, Rinn JL, and Pachter L. 2012. Differential gene and transcript expression analysis of RNA-seq experiments with TopHat and Cufflinks. Nature Protocols 7:562-578 DOI 10.1038/nprot.2012.016

Varotto S, Locatelli S, Canova S, Pipal A, Motto M, and Rossi V. 2003. Expression profile and cellular localization of maize Rpd3-type histone deacetylases during plant development. Plant Physiology 133:606-617 DOI 10.1104/pp.103.025403

Wang SH, Lim JH, Kim SS, Cho SH, Yoo SC, Koh HJ, Sakuraba Y, and Paek NC. 2015. Mutation of SPOTTED LEAF3 (SPL3) impairs abscisic acid-responsive signalling and delays leaf senescence in rice. Journal of Experimental Botany 66:7045-7059 DOI $10.1093 / \mathrm{jxb} / \mathrm{erv} 401$ 
525 Yang H, Liu X, Xin M, Du J, Hu Z, Peng H, Rossi V, Sun Q, Ni Z, and Yao Y. 2016. Genome-

526

527

528

529

530

531

532

533

534

535

536

537

538

539

540

541

542

543

544

545

546

547

548

549

550

551 wide mapping of targets of maize histone deacetylase HDA101 reveals Its function and regulatory mechanism during seed development. The Plant Cell 28:629-645 DOI $10.1105 /$ tpc. 15.00691

Yang XJ, and Seto E. 2008. The Rpd3/Hda1 family of lysine deacetylases: from bacteria and yeast to mice and men. Nature Reviews Molecular Cell Biology 9:206-218 DOI $10.1038 / \mathrm{nrm} 2346$

Yu CW, Liu X, Luo M, Chen C, Lin X, Tian G, Lu Q, Cui Y, and Wu K. 2011. HISTONE DEACETYLASE6 interacts with FLOWERING LOCUS D and regulates flowering in Arabidopsis. Plant Physiology 156:173-184 DOI 10.1104/pp.111.174417

Yuan L, Liu X, Luo M, Yang S, and Wu K. 2013. Involvement of histone modifications in plant abiotic stress responses. Journal of Integrative Plant Biology 55:892-901 DOI 10.1111/jipb.12060

Zhao J, Li M, Gu D, Liu X, Zhang J, Wu K, Zhang X, Teixeira da Silva JA, and Duan J. 2016. Involvement of rice histone deacetylase HDA705 in seed germination and in response to ABA and abiotic stresses. Biochemical and Biophysical Research Communications 470:439-444 DOI 10.1016/j.bbrc.2016.01.016

Zhao J, Zhang J, Zhang W, Wu K, Zheng F, Tian L, Liu X, and Duan J. 2014a. Expression and functional analysis of the plant-specific histone deacetylase HDT701 in rice. Frontiers in Plant Science 5:764 DOI 10.3389/fpls.2014.00764

Zhao L, Lu J, Zhang J, Wu PY, Yang S, and Wu K. 2014b. Identification and characterization of histone deacetylases in tomato (Solanum lycopersicum). Frontiers in Plant Science 5:760 DOI 10.3389/fpls.2014.00760

Zhou C, Labbe H, Sridha S, Wang L, Tian L, Latoszek-Green M, Yang Z, Brown D, Miki B, and Wu K. 2004. Expression and function of HD2-type histone deacetylases in Arabidopsis development. The Plant Journal 38:715-724 DOI 10.1111/j.1365-313X.2004.02083.x

Zhou C, Zhang L, Duan J, Miki B, and Wu K. 2005. HISTONE DEACETYLASE19 is involved 
552 in jasmonic acid and ethylene signaling of pathogen response in Arabidopsis. The Plant Cell 17:1196-1204 DOI 10.1105/tpc.104.028514

554 Zhu Z, An F, Feng Y, Li P, Xue L, A M, Jiang Z, Kim JM, To TK, Li W, Zhang X, Yu Q, Dong Z, Chen WQ, Seki M, Zhou JM, and Guo H. 2011. Derepression of ethylene-stabilized transcription factors (EIN3/EIL1) mediates jasmonate and ethylene signaling synergy in Arabidopsis. Proceedings of the National Academy of Sciences of the United States of America 108:12539-12544 DOI 10.1073/pnas.1103959108 
Figure 1

Figure 1. Phylogenetic relationship of HDAC gene family among maize, rice, and Arabidopsis. Multiple sequences alignment and phylogenetic tree construction were performed using MEGA7.0.

The value at the nodes represents bootstrap values from 1000 replicates. Different groups are shown by different colors.

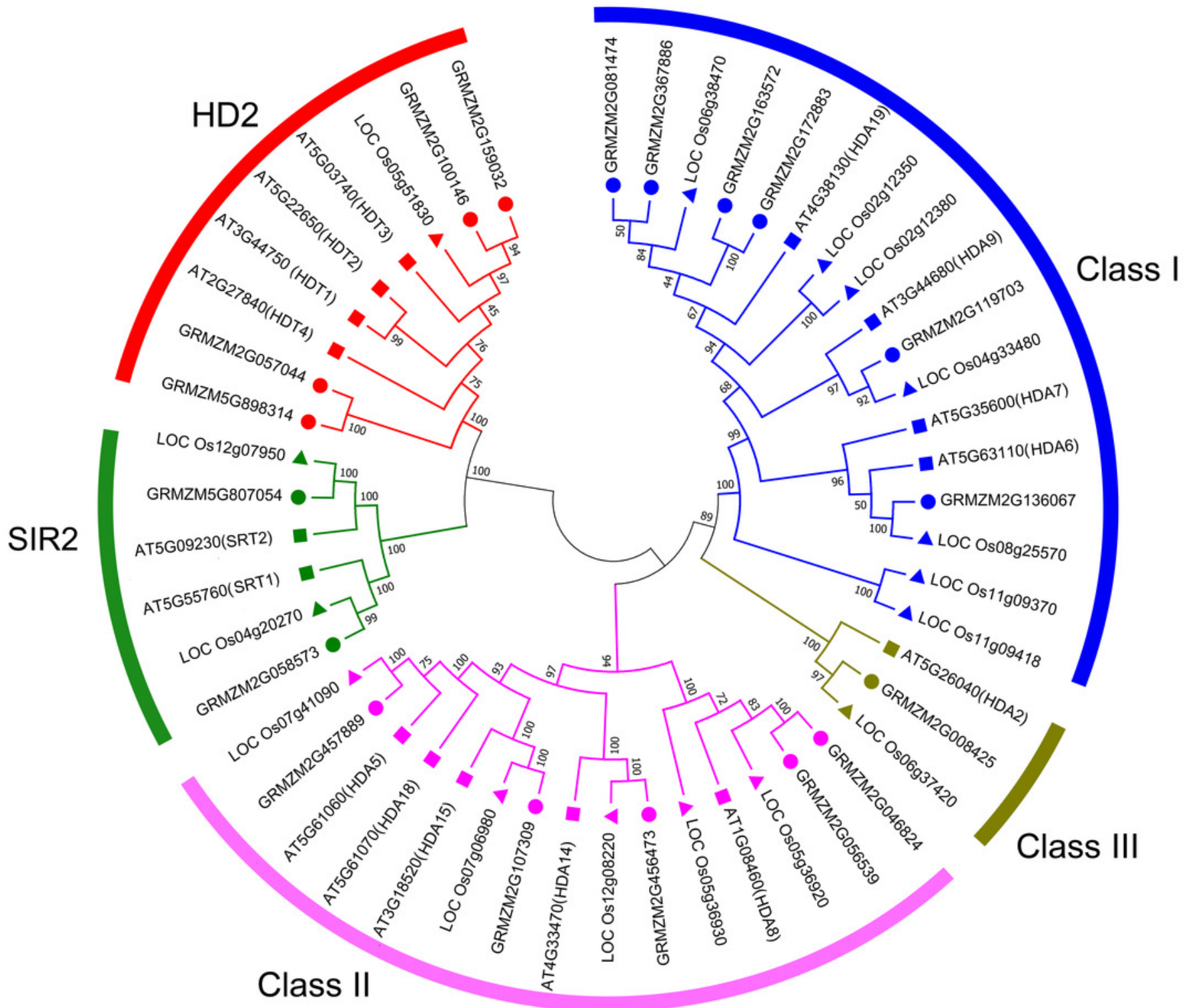




\section{Figure 2}

Figure 2. Phylogenetic analysis and domain architecture of ZmHDACs. Construction of phylogenetic tree based on ZmHDACs amino acid sequences. Conserved domains of ZmHDACs were identified by Pfam and SMART.

Different domains are indicated by different colors. The lengths of the domains in each protein are proportional. The NJ phylogenetic tree of ZmHDACs protein sequences was constructed using 1000 bootstrap replicates by MEGA 7.0. HDAC, Histone deacetylase domain; Sir2, Sir2 catalytic domain; ZnF_C2H2, Zinc finger $\mathrm{C} 2 \mathrm{H} 2$ type domain.

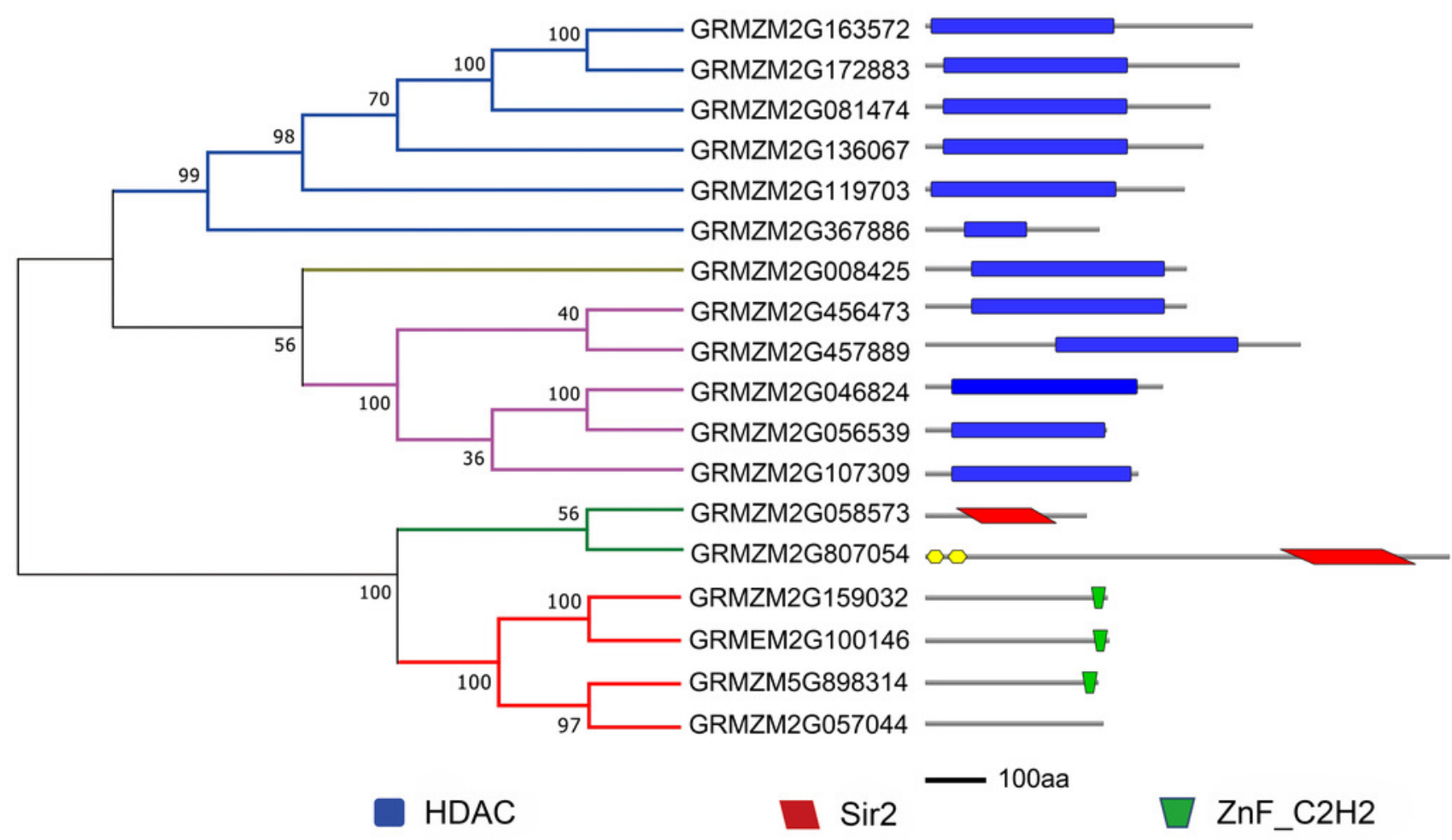




\section{Figure 3}

Figure 3. Heatmaps representing the expression profiles of ZmHDAC genes in several tissues. Tissue-specific expression patterns of ZmHDAC genes associated with 22 different tissues or organs.

The color scale on the right indicates expression values, with blue denoting high expression level and yellow representing low expression level.

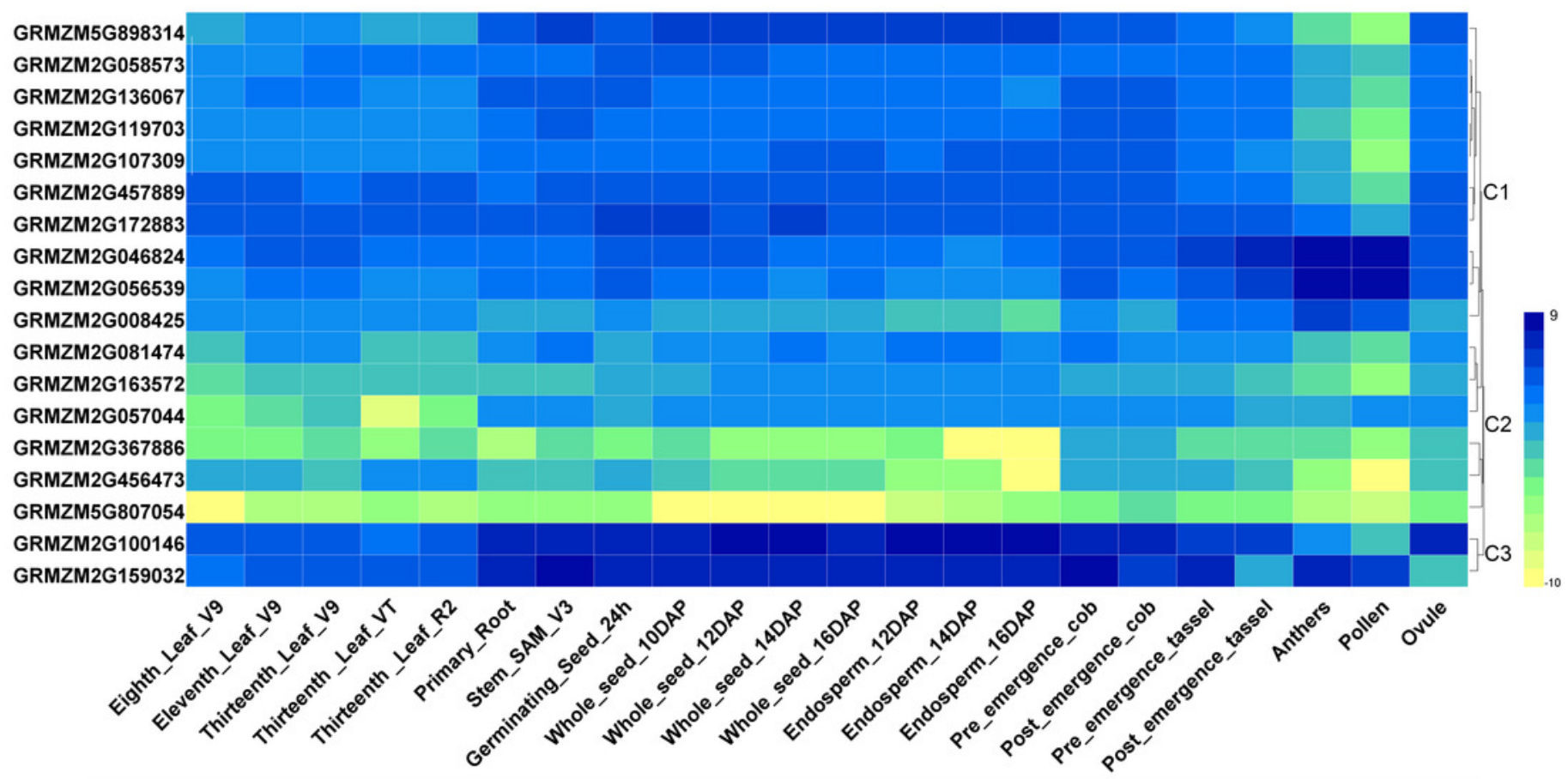




\section{Figure 4}

Figure 4. Expression patterns of ZmHDAC genes under F. verticillioides infection. The color scale on the right indicates fold changes (inoculated/mock), with blue denoting high fold change and yellow showing low fold change.

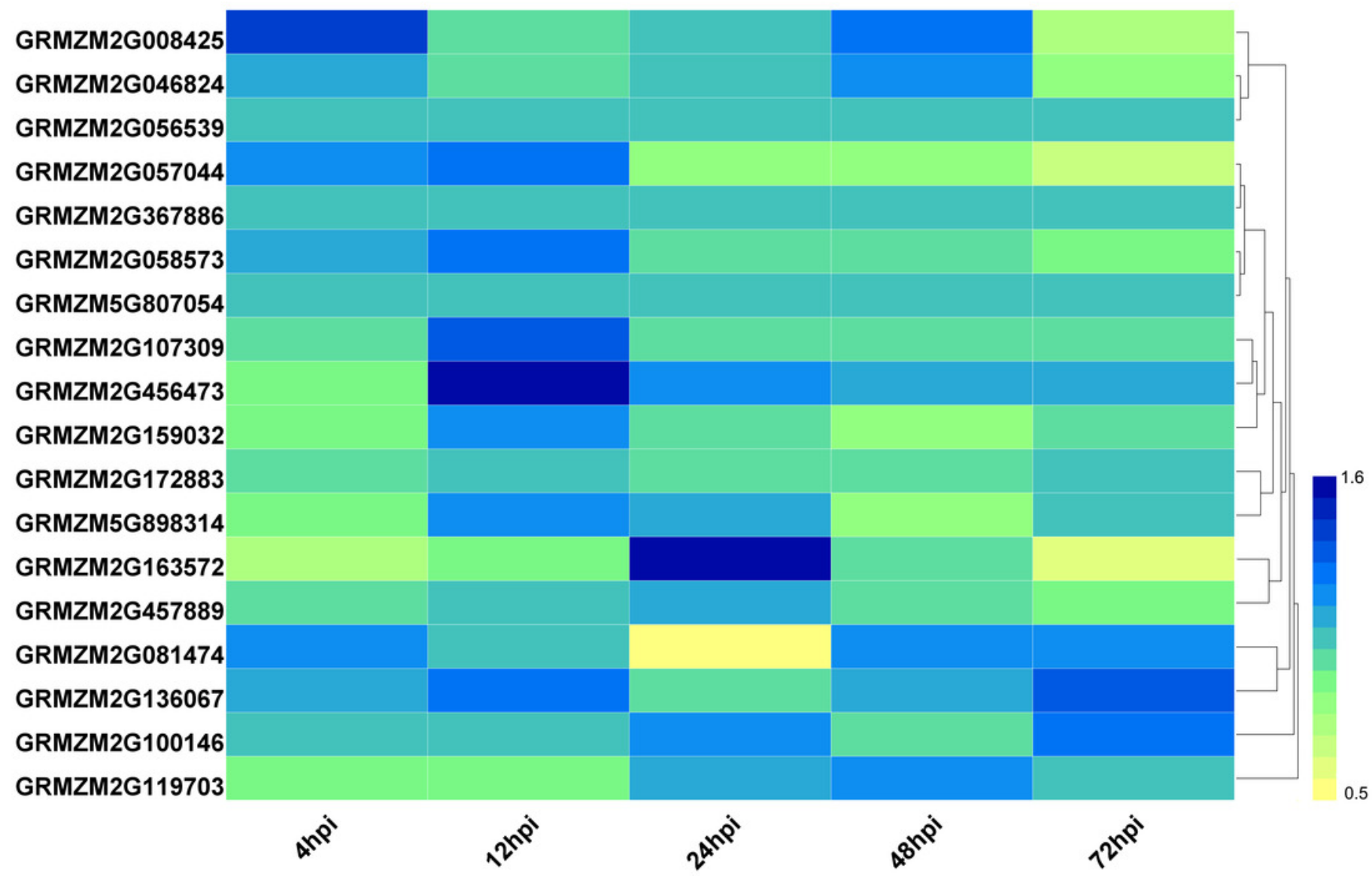


Figure 5

Figure 5. Expression patterns of ZmHDAC genes in seedling leaf under MejA treatment. qRT-PCR was performed using gene-specific primers. Data are the mean \pm SEM of three independent experiments.
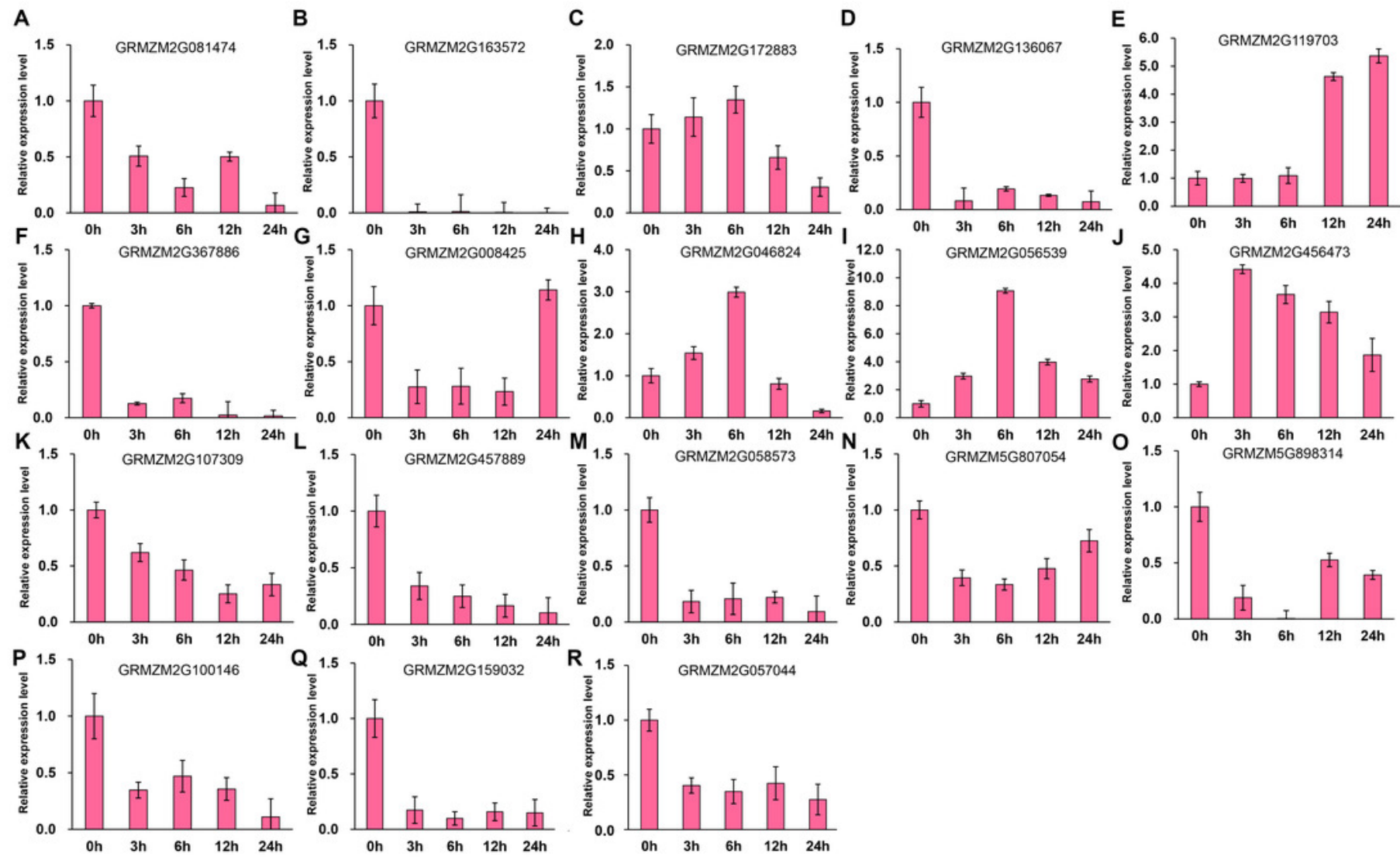
Figure 6

Figure 6. Expression patterns of ZmHDAC genes in seedling leaf under SA treatment. qRT-PCR was performed using gene-specific primers. Data are the mean \pm SEM of three independent experiments.
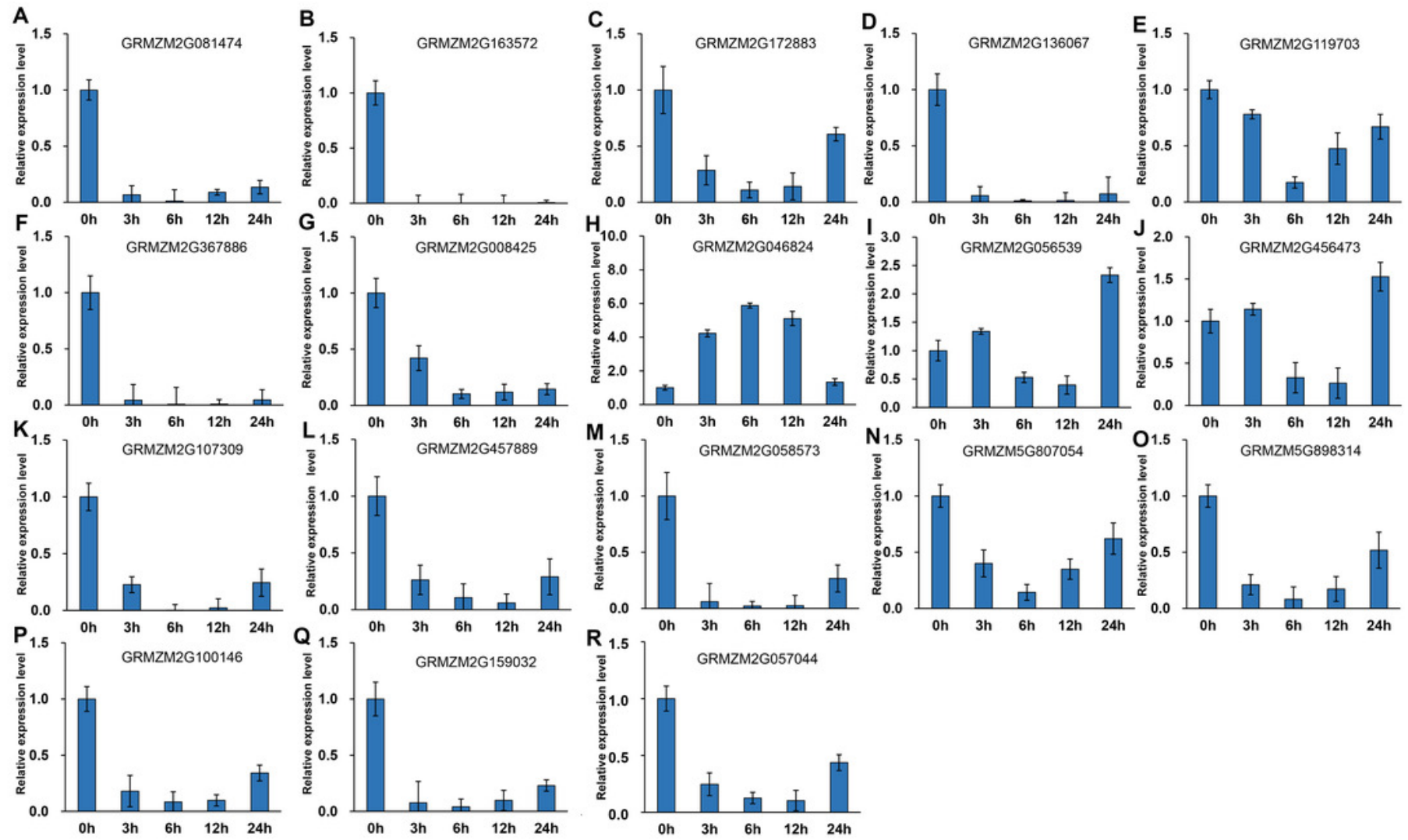


\section{Figure 7}

Figure 7. Expression patterns of ZmHDAC genes under heat, cold, salt, UV, and drought stresses. The color scale on the right indicates expression values, with blue denoting high expression level and yellow representing low expression level.

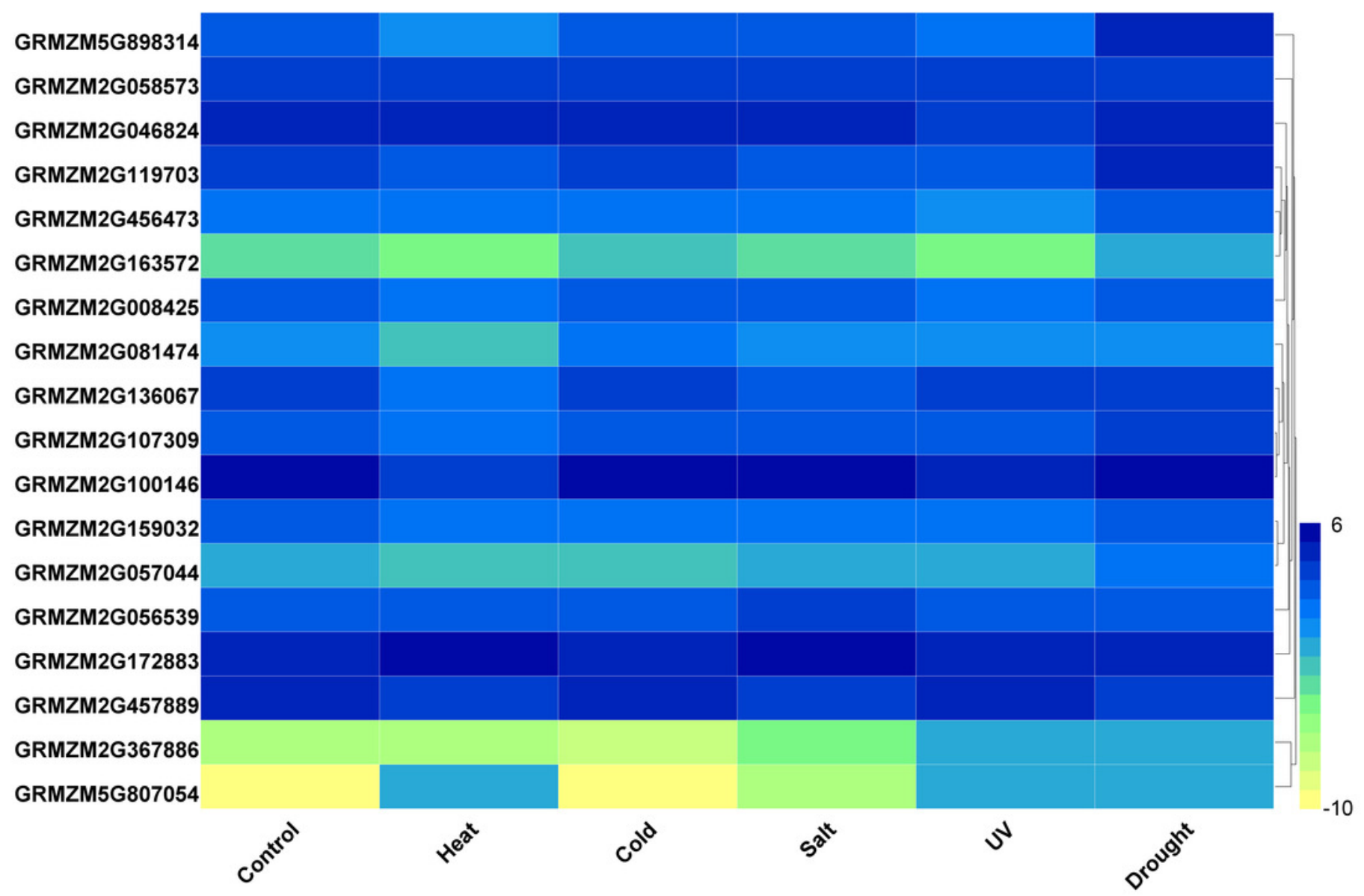


Figure 8

Figure 8. Immunoblot for the detection of $\mathrm{H} 3 \mathrm{~K} 9 \mathrm{ac}$ and $\mathrm{H} 4 \mathrm{~K} 5 \mathrm{ac}$ levels in seedling of the B73 inbred line under cold and heat treatments. Immunoblot with anti-H3 antibody was used as a loading control. CK mean the control treatment temperature $\left(25^{\circ} \mathrm{C}\right)$, cold

\section{H3K9ac}

1.00

0.95

1.45

\section{H4K5ac}

0.89

1.34

H3 
Table $\mathbf{1}$ (on next page)

HDAC genes in maize. 
Table 1. HDAC genes information in maize

\begin{tabular}{|c|c|c|c|c|c|c|c|c|c|}
\hline & Gene ID & Gene name & $\mathrm{Chr}$ & Start & End & CDS(bp) & AA & MW(Da) & $\mathrm{pI}$ \\
\hline \multirow{12}{*}{ RPD3/HDA1 } & GRMZM2G163572 & & $\operatorname{chr} 5$ & 156416899 & 156406486 & 1515 & 504 & 56689.26 & 5.65 \\
\hline & GRMZM2G172883 & HDA101 & $\operatorname{chr} 4$ & 231342828 & 231335846 & 1551 & 516 & 57803.5 & 5.43 \\
\hline & GRMZM2G081474 & & chr9 & 84349561 & 84342406 & 1410 & 469 & 52779.31 & 5.73 \\
\hline & GRMZM2G119703 & HDA102 & $\operatorname{chr} 2$ & 59103871 & 59090885 & 1092 & 363 & 41405.76 & 4.77 \\
\hline & GRMZM2G136067 & HDA108 & $\operatorname{chr} 4$ & 65988818 & 65983218 & 1377 & 458 & 50940.17 & 5.43 \\
\hline & GRMZM2G367886 & & chr6 & 168403680 & 168402676 & 501 & 166 & 18907.73 & 9.17 \\
\hline & GRMZM2G456473 & & $\operatorname{chr} 3$ & 182718225 & 182648800 & 1152 & 383 & 43341.55 & 8.91 \\
\hline & GRMZM2G457889 & HDA109 & $\operatorname{chr} 2$ & 210298363 & 210311868 & 2103 & 700 & 76544.96 & 5.54 \\
\hline & GRMZM2G056539 & & chr8 & 99989295 & 99984845 & 900 & 299 & 31909.77 & 5.33 \\
\hline & GRMZM2G046824 & & chr8 & 99109552 & 99100490 & 834 & 277 & 29263.26 & 6.1 \\
\hline & GRMZM2G107309 & HDA110 & $\operatorname{chr} 7$ & 7285361 & 7273053 & 1860 & 619 & 66186.64 & 5.74 \\
\hline & GRMZM2G008425 & & $\operatorname{chr} 9$ & 80424376 & 80418132 & 1056 & 351 & 38784.55 & 6.71 \\
\hline \multirow{2}{*}{ SIR2 } & GRMZM2G058573 & SRT101 & $\operatorname{chr} 10$ & 125947058 & 125922937 & 1314 & 437 & 49050.03 & 9.31 \\
\hline & GRMZM5G807054 & & $\operatorname{chr} 10$ & 10823442 & 10811717 & 1056 & 351 & 39188.5 & 8.91 \\
\hline \multirow{4}{*}{ HD2 } & GRMZM2G057044 & HDT101/HDA106 & $\operatorname{chr} 3$ & 164420298 & 164429357 & 882 & 293 & 31810.94 & 4.29 \\
\hline & GRMZM2G100146 & HDT102/HDA103 & $\operatorname{chr} 8$ & 135667488 & 135662882 & 906 & 301 & 32402.15 & 4.61 \\
\hline & GRMZM2G159032 & HDT103/HDA105 & chr6 & 161429635 & 161426908 & 903 & 300 & 32501.43 & 4.69 \\
\hline & GRMZM5G898314 & HDT104 & chr8 & 162666204 & 162662841 & 858 & 285 & 30435.21 & 4.75 \\
\hline
\end{tabular}

3 Start: The first physical position of the gene on the chromosome; End: The last physical position of the gene on the chromosome; CDS: Coding sequence;

4 AA: Amino acid length; MW: molecular weight; pI: Isoelectric point 